Article

\title{
On An Open Question in Controlled Rectangular $b$-Metric Spaces
}

\author{
Reny George ${ }^{1,2, *}$, Abdelkader Belhenniche ${ }^{3,4}$, Sfya Benahmed ${ }^{3}$, , Zoran D. Mitrović ${ }^{5, *} *$, \\ Nabil Mlaiki ${ }^{6}$ (i) and Liliana Guran ${ }^{7}$ \\ 1 Department of Mathematics, College of Science and Humanities in Alkharj, \\ Prince Sattam bin Abdulaziz University, Al-Kharj 11942, Saudi Arabia \\ 2 Department of Mathematics and Computer Science, St. Thomas College, Bhilai, Chhattisgarh 491022, India \\ 3 École Nationale Polytechnique d'Oran Maurice Audin, School in Oran, B.P 1523 El M'naouer, \\ Oran 31000, Algeria; belhennicheabdelkader@yahoo.com (A.B.); sfyabenahmed@yahoo.fr (S.B.) \\ 4 SYSTEC - Research Center for Systems and Technologies, Department of Electrical Engineering, \\ Faculty of Engineering, Porto University, 4200-465 Porto, Portugal \\ 5 Faculty of Electrical Engineering, University of Banja Luka, Patre 5, \\ 78000 Banja Luka, Bosnia and Herzegovina \\ 6 Department of Mathematics and General Sciences, Prince Sultan University Riyadh, \\ Riyadh 11586, Saudi Arabia; nmlaiki@psu.edu.sa \\ 7 Department of Pharmaceutical Sciences, Faculty of Pharmacy, Vasile Goldiş Western University Arad, \\ L. Rebreanu Street, no. 86, 310048 Arad, Romania; guran.liliana@uvvg.ro \\ * Correspondence: r.kunnelchacko@psau.edu.sa or renygeorge02@yahoo.com (R.G.); \\ zoran.mitrovic@etf.unibl.org (Z.D.M.)
}

Received: 12 November 2020; Accepted: 15 December 2020; Published: 18 December 2020

\begin{abstract}
In this paper, we give an affirmative answer to an open question posed recently by Mlaiki et al. As a consequence of our results, we get some known results in the literature. We also give an application of our results to the existence of a solution of nonlinear fractional differential equations.
\end{abstract}

Keywords: rectangular $b$-metric spaces; controlled rectangular $b$-metric spaces; fixed point

\section{Introduction}

Fréchet [1] introduced the axiomatic form of distance as L-space. Hausdorff [2] re-defined it as a metric space. In 1922, Banach [3] proved the existence and uniqueness of a fixed point for self-contractive mappings in a complete metric space. Inspired by the wide applications of Banach's result, numerous extensions and generalizations of it appeared in the literature. One direction of such generalizations was by generalizing the concept of a metric space itself. Bakhtin [4], Branciari [5], George et al. [6], Mlaiki et al. [7], Abdeljawad et al. [8,9], and Shatanawi et al. [10] introduced the notion of a $b$-metric, rectangular metric, rectangular $b$-metric, controlled metric, double controlled metric, extended Branciari $b$-distance, and extended $b$-metric, respectively (see also [11-13]). The concept of a controlled rectangular $b$-metric space was introduced in Mlaiki et al. [14]. The following two theorems are the main results in [14].

Theorem 1. Let $\left(X, D_{\xi}\right)$ be a $D_{\xi}$-complete, controlled, rectangular b-metric space, $T: X \rightarrow X$ be a self map on $X$. If there exists $0<\delta<1$, such that

$$
D_{\mathcal{\zeta}}(T z, T t) \leq \delta D_{\mathcal{\zeta}}(z, t),
$$


for all $z, t \in X$, and

$$
\sup _{m>1} \lim _{n \rightarrow \infty} \xi\left(x_{n}, x_{n+1}, x_{n+2}, x_{m}\right) \leq \frac{1}{\delta},
$$

then $T$ has a unique fixed point in $X$.

Remark 1. Note that, from Theorem 1 we obtain theorem 2.1 in [6].

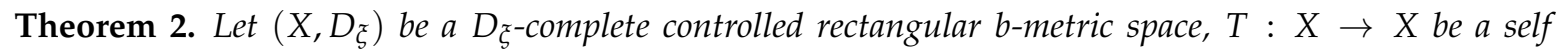
map on $X$. If

$$
D_{\xi}(T z, T t) \leq \delta\left[D_{\xi}(z, T z)+D_{\xi}(t, T t)\right]
$$

for all $z, t \in E$, wher $\delta \in(0,1)$. Also, if

$$
\sup _{m>1} \lim _{n \rightarrow \infty} \xi\left(x_{n}, x_{n+1}, x_{n+2}, x_{m}\right) \leq \frac{1}{\delta}
$$

and for all $u, v \in X$ we have

$$
\lim _{n \rightarrow \infty} \xi\left(u, v, x_{n}, x_{n+1}\right) \leq 1
$$

then $T$ has a unique fixed point in $X$.

Mlaiki et al. in the paper [14] gave the following open question.

Question 1. Let $\left(X, D_{\xi}\right)$ be a controlled rectangular b-metric space, and $T: X \rightarrow X$ be a self map on $X$. Assume that for all distinct $u, v, T u, T v \in X$ there exists $\delta \in(0,1)$ such that

$$
D_{\xi}(T u, T v) \leq \delta \xi(u, v, T u, T v) D_{\xi}(u, v) .
$$

What are the other hypotheses we should add so that T has a unique fixed point in the whole space X?

The aim of this work is to provide an answer to the above question by providing other hypotheses required to prove the existence of a unique fixed point of the mapping $T$ given in Question 1 . We also provide a short proof of Theorem 1, and we replace condition (1) with

$$
\sup _{m, n, k \in \mathbb{N}} \xi\left(x_{m}, x_{n}, x_{m+k}, x_{n+k}\right)<+\infty
$$

Also, we show that condition (2) is redundant and that condition (3) can be relaxed.

\section{Preliminaries}

Definition 1 ([14]). Let $X$ be a non-empty set, $\xi: X^{4} \rightarrow[1, \infty)$ and $D_{\xi}: X^{2} \rightarrow[0, \infty)$ be given mappings. We say that $\left(X, D_{\xi}\right)$ is a controlled rectangular b-metric space (or in short CRb-MS) if, for all distinct $c, d, e, f \in X$, we have

1. $D_{\xi}(c, d)=0$ if and only if $c=d$;

2. $D_{\xi}(c, d)=D_{\xi}(d, c)$;

3. $\quad D_{\xi}(c, d) \leq \xi(c, d, e, f)\left[D_{\xi}(c, e)+D_{\xi}(e, f)+D_{\xi}(f, d)\right]$.

Further, through the paper we will assume that $\left(X, D_{\xi}\right)$ is a CRb-MS. The following definition presents the topology of this space.

Definition 2 ([14]).

1. A sequence $\left\{a_{n}\right\}$ is called $D_{\xi}$-convergent in $\left(X, D_{\xi}\right)$, if there exists $a \in X$ such that $\lim _{n \rightarrow \infty} D_{\xi}\left(a_{n}, a\right)=0$. 
2. A sequence $\left\{a_{n}\right\}$ in $\left(X, D_{\xi}\right)$ is called $D_{\xi^{-}}$Cauchy if $\lim _{n, m \rightarrow \infty} D_{\tilde{\xi}}\left(a_{n}, a_{m}\right)=0$.

3. $\left(X, D_{\xi}\right)$ is called $D_{\xi}$-complete if every $D_{\xi}$-Cauchy sequence $\left\{a_{n}\right\}$ in $X$ is convergent in $X$.

4. For any $c \in X$ and $\eta>0$, we define an open ball in $\left(X, D_{\xi}\right)$ with center $c$ and radius $\eta$ by $B_{\xi}(c, \eta)=\left\{d \in X \mid D_{\xi}(c, d)<\eta\right\}$.

Remark 2. Note that the condition (4) gives us the possibility to obtain, as a consequence of our result, a series of known results in a b-metric space, rectangular metric space, $b$-rectangular metric space, extended b-metric space, and extended b-rectangular metric space. Additionally, rectangular metric spaces (see Branciari in [5]), rectangular b-metric spaces (see George et al. [6]), and extended Branciari b-distance spaces (see Abdeljawad et al. in [9]) are all CRb-MS. (The converse is not necessarily true (see Example 1, [14]).

Remark 3. In this paper, we consider that the sequence $\left\{x_{n}\right\}$ is defined by $x_{n}=T x_{n-1}, n \in \mathbb{N}$, where $x_{0} \in X$.

\section{An Answer to an Open Question}

Theorem 3. Let $T: X \rightarrow X$ be a mapping on $D_{\tilde{\xi}}$-complete $C R b-M S\left(X, D_{\tilde{\xi}}\right)$. Assume that

$$
D_{\xi}(T u, T w) \leq \delta \xi(u, w, T u, T w) D_{\xi}(u, w)
$$

for all distinct $u, w, T u, T w \in X$, where $\delta \in[0,1)$. If

$$
\sup _{m, n, k \in \mathbb{N}} \prod_{i=1}^{k} \xi\left(x_{m+k-i}, x_{n+k-i}, x_{m+k+1-i}, x_{n+k+1-i}\right)<+\infty
$$

and

$$
\sup _{m, n, k \in \mathbb{N}} \xi\left(x_{m}, x_{n}, x_{m+k}, x_{n+k}\right)<+\infty,
$$

then $T$ has a unique fixed point in $X$.

Proof. Step 1. Let $\left\{x_{n}\right\}$ be a sequence defined as in Remark 3. If $x_{n}=x_{n+1}$ then $x_{n}$ is a fixed point of $T$ and we are done. So suppose that $x_{n} \neq x_{n+1}$ for all $n \geq 0$. Then $x_{n} \neq x_{n+k}$ for all $n \geq 0, k \geq 1$. Then, we assume $x_{n} \neq x_{m}$ for all distinct $n, m \in \mathbb{N}$. Note that $x_{n+k} \neq x_{m+k}$ for all distinct $n, m \in \mathbb{N}$ $x_{n+k}, x_{m+k} \in X \backslash\left\{x_{n}, x_{m}\right\}$.

Step 2. From condition (6), we conclude that there exists $M>0$, such that

$$
\prod_{i=0}^{k} \xi\left(x_{m+k-i}, x_{n+k-i}, x_{m+k+1-i}, x_{n+k+1-i}\right) \leq M
$$

for all $m, n, k \in \mathbb{N}$. Since $\delta \in[0,1)$, we conclude that there exists $n_{1} \in \mathbb{N}$, such that

$$
\delta^{n_{1}} M<1
$$

Let $k, m, m \in \mathbb{N}$, such that

$$
\min \{k, m, n\} \geq n_{1} \text {. }
$$

Now, from Condition 3 of Definition 1, we have

$$
\begin{aligned}
D_{\xi}\left(x_{m}, x_{n}\right) & \leq \xi\left(x_{m}, x_{n}, x_{m+k}, x_{n+k}\right)\left[D_{\xi}\left(x_{m}, x_{m+k}\right)\right. \\
& \left.+D_{\xi}\left(x_{m+k}, x_{n+k}\right)+D_{\xi}\left(x_{n+k}, x_{n}\right)\right] .
\end{aligned}
$$


Next, from Condition (5), we have

$$
\begin{aligned}
D_{\xi}\left(x_{m}, x_{m+k}\right) \leq & \delta \xi\left(x_{m-1}, x_{m+k-1}, x_{m}, x_{m+k}\right) D_{\xi}\left(x_{m-1}, x_{m+k-1}\right) \\
\leq & \delta^{2} \xi\left(x_{m-1}, x_{m+k-1}, x_{m}, x_{m+k}\right) \\
& \xi\left(x_{m-2}, x_{m+k-2}, x_{m-1}, x_{m+k-1}\right) D_{\xi}\left(x_{m-2}, x_{m+k-2}\right) \\
\vdots & \delta^{m} \prod_{i=1}^{m} \xi\left(x_{m-i}, x_{m+k-i}, x_{m+1-i}, x_{m+k+1-i}\right) D_{\xi}\left(x_{0}, x_{k}\right) .
\end{aligned}
$$

Similar as in (12), we get

$$
D_{\xi}\left(x_{n}, x_{n+k}\right) \leq \delta^{n} \prod_{i=1}^{n} \xi\left(x_{n-i}, x_{n+k-i}, x_{n+1-i}, x_{n+k+1-i}\right) D_{\xi}\left(x_{0}, x_{k}\right)
$$

and

$$
D_{\xi}\left(x_{m+k}, x_{n+k}\right) \leq \delta^{k} \prod_{i=1}^{k} \xi\left(x_{m+k-i}, x_{n+k-i}, x_{m+k+1-i}, x_{n+k+1-i}\right) D_{\xi}\left(x_{m}, x_{n}\right) .
$$

Let

$$
\begin{aligned}
P_{m, n} & =\prod_{i=1}^{k} \xi\left(x_{m+k-i}, x_{n+k-i}, x_{m+k+1-i}, x_{n+k+1-i}\right), \\
P_{m} & =\prod_{i=1}^{m} \xi\left(x_{m-i}, x_{m+k-i}, x_{m+1-i}, x_{m+k+1-i}\right), \\
P_{n} & =\prod_{i=1}^{n} \xi\left(x_{n-i}, x_{n+k-i}, x_{n+1-i}, x_{n+k+1-i}\right) .
\end{aligned}
$$

From (11)-(14), we obtain

$$
\begin{aligned}
D_{\xi}\left(x_{m}, x_{n}\right) & \leq \xi\left(x_{m}, x_{n}, x_{m+k}, x_{n+k}\right)\left[\delta^{m} P_{m} D_{\xi}\left(x_{0}, x_{k}\right)\right. \\
& \left.+\delta^{k} P_{m, n} D_{\xi}\left(x_{m}, x_{n}\right)+\delta^{n} P_{n} D_{\xi}\left(x_{k}, x_{0}\right)\right] .
\end{aligned}
$$

Due to the conditions (7) and (9), we have

$$
D_{\xi}\left(x_{m}, x_{n}\right) \leq \xi\left(x_{m}, x_{n}, x_{m+k}, x_{n+k}\right) \frac{\left(\delta^{m} P_{m}+\delta^{n} P_{n}\right) D_{\xi}\left(x_{0}, x_{k}\right)}{1-\delta^{k} \xi\left(x_{m}, x_{n}, x_{m+k}, x_{n+k}\right) P_{m, n}} .
$$

Using condition (6), we obtain that $\left\{x_{n}\right\}$ is a $D_{\xi}$-Cauchy. Thus, there exists $x^{*} \in X$ such that

$$
\lim _{n \rightarrow \infty} x_{n}=x^{*}
$$

We have

$$
\begin{aligned}
D_{\xi}\left(x^{*}, T x^{*}\right) & \leq \xi\left(x^{*}, T x^{*}, x_{n}, x_{n+1}\right)\left[D_{\xi}\left(x^{*}, x_{n}\right)+D_{\xi}\left(x_{n}, x_{n+1}\right)+D_{\xi}\left(x_{n+1}, T x^{*}\right)\right] \\
& \leq \xi\left(x^{*}, T x^{*}, x_{n}, x_{n+1}\right)\left[D_{\xi}\left(x^{*}, x_{n}\right)+D_{\xi}\left(x_{n}, x_{n+1}\right)+D_{\xi}\left(T x_{n}, T x^{*}\right)\right] \\
& \leq \xi\left(x^{*}, T x^{*}, x_{n}, x_{n+1}\right)\left[D_{\xi}\left(x^{*}, x_{n}\right)+D_{\xi}\left(x_{n}, x_{n+1}\right)+\right. \\
& \left.\delta \xi\left(x^{*}, T x^{*}, x_{n}, x_{n+1}\right) D_{\xi}\left(x_{n}, x^{*}\right)\right] .
\end{aligned}
$$

Since $\lim _{n \rightarrow \infty} D_{\xi}\left(x^{*}, x_{n}\right)=0$ and $\lim _{n \rightarrow \infty} D_{\xi}\left(x_{n}, x_{n+1}\right)=0$, we conclude $D_{\xi}\left(x^{*}, T x^{*}\right)=0$, i.e $T x^{*}=x^{*}$.

Step 3. Uniqueness. Let $y^{*} \in X$ such that $T y^{*}=y^{*}$. Then we have

$$
D_{\xi}\left(x^{*}, y^{*}\right) \leq \xi\left(x^{*}, y^{*}, x_{n}, x_{n+1}\right)\left[D_{\xi}\left(x^{*}, x_{n}\right)+D_{\xi}\left(x_{n}, x_{n+1}\right)+D_{\xi}\left(x_{n+1}, y^{*}\right)\right] .
$$


Since $\lim _{n \rightarrow \infty} D_{\tilde{\zeta}}\left(x^{*}, x_{n}\right)=0, \lim _{n \rightarrow \infty} D_{\tilde{\zeta}}\left(x_{n}, x_{n+1}\right)=0, \lim _{n \rightarrow \infty} D_{\tilde{\zeta}}\left(x_{n+1}, y^{*}\right)=0$ we obtain $D_{\tilde{\zeta}}\left(x^{*}, y^{*}\right)=0$. Thus, $x^{*}=y^{*}$.

\section{On Fixed Point Theorems of Banach and Kannan}

Now, we give another variant of the Theorem 1.

Theorem 4. Let $T: X \rightarrow X$ be a mapping on $D_{\xi}$-complete $C R b-M S\left(X, D_{\xi}\right)$. Suppose that for all distinct $u, w, T u, T w \in X$, there exists $\delta \in[0,1)$, such that

$$
D_{\mathcal{\zeta}}(T u, T w) \leq \delta D_{\xi}(u, w)
$$

and

$$
\sup _{m, n, k \in \mathbb{N}} \xi\left(x_{m}, x_{n}, x_{m+k}, x_{n+k}\right)<+\infty .
$$

Then, $T$ has a unique fixed point in X.

Proof. Step 1. Same as in Theorem 3.

Step 2. From condition (19), we obtain that there exists $M>0$ such that

$$
\xi\left(x_{m}, x_{n}, x_{m+k}, x_{n+k}\right) \leq M,
$$

for all $m, n, k \in \mathbb{N}$. Since $\delta \in[0,1)$ we conclude that there exists $n_{1} \in \mathbb{N}$ such that

$$
\delta^{n_{1}} M<1
$$

Let $k, m, m \in \mathbb{N}$ such that

$$
\min \{k, m, n\} \geq n_{1}
$$

Next, we have

$$
\begin{aligned}
D_{\xi}\left(x_{m}, x_{n}\right) & \leq \xi\left(x_{m}, x_{n}, x_{m+k}, x_{n+k}\right)\left[D_{\xi}\left(x_{m}, x_{m+k}\right)\right. \\
& \left.+D_{\xi}\left(x_{m+k}, x_{n+k}\right)+D_{\xi}\left(x_{n+k}, x_{n}\right)\right] .
\end{aligned}
$$

Next, from condition (18), we have

$$
\begin{aligned}
D_{\mathcal{\xi}}\left(x_{m}, x_{m+k}\right) & \leq \delta D_{\xi}\left(x_{m-1}, x_{m+k-1}\right) \\
& \vdots \\
& \leq \delta^{m} D_{\mathcal{\zeta}}\left(x_{0}, x_{k}\right) .
\end{aligned}
$$

Additionally, as in (24), we obtain

$$
D_{\xi}\left(x_{n}, x_{n+k}\right) \leq \delta^{n} D_{\xi}\left(x_{0}, x_{k}\right)
$$

and

$$
D_{\xi}\left(x_{m+k}, x_{n+k}\right) \leq \delta^{k} D_{\xi}\left(x_{m}, x_{n}\right) .
$$

So, we obtain

$$
D_{\xi}\left(x_{m}, x_{n}\right) \leq \xi\left(x_{m}, x_{n}, x_{m+k}, x_{n+k}\right) \frac{\left(\delta^{m}+\delta^{n}\right) D_{\xi}\left(x_{0}, x_{k}\right)}{1-\delta^{k} \xi\left(x_{m}, x_{n}, x_{m+k}, x_{n+k}\right)} .
$$


Next, using condition (19), we obtain that $\left\{x_{n}\right\}$ is a $D_{\xi}$-Cauchy. Therefore, there exists $x^{*} \in X$ such that

$$
\lim _{n \rightarrow \infty} x_{n}=x^{*}
$$

Now let us prove that $x^{*}$ is a fixed point. We have

$$
\begin{aligned}
D_{\mathcal{\zeta}}\left(x^{*}, T x^{*}\right) & \leq \xi\left(x^{*}, T x^{*}, x_{n}, x_{n+1}\left[D_{\mathcal{\zeta}}\left(x^{*}, x_{n}\right)+D_{\mathcal{\zeta}}\left(x_{n}, x_{n+1}\right)+D_{\xi}\left(x_{n+1}, T x^{*}\right)\right]\right. \\
& \leq \xi\left(x^{*}, T x^{*}, x_{n}, x_{n+1}\right)\left[D_{\mathcal{\zeta}}\left(x^{*}, x_{n}\right)+D_{\xi}\left(x_{n}, x_{n+1}\right)+D_{\xi}\left(T x_{n}, T x^{*}\right)\right] \\
& \leq \xi\left(x^{*}, T x^{*}, x_{n}, x_{n+1}\right)\left[D_{\mathcal{\xi}}\left(x^{*}, x_{n}\right)+D_{\xi}\left(x_{n}, x_{n+1}\right)+\right. \\
& \left.\delta D_{\xi}\left(x_{n}, x^{*}\right)\right] .
\end{aligned}
$$

Since

$$
\lim _{n \rightarrow \infty} D_{\tilde{\xi}}\left(x^{*}, x_{n}\right)=0, \lim _{n \rightarrow \infty} D_{\mathcal{\xi}}\left(x_{n}, x_{n+1}\right)=0,
$$

we conclude

$$
D_{\xi}\left(x^{*}, T x^{*}\right)=0 .
$$

Step 3. Uniqueness. Let $y^{*}$ be another fixed point of $T$. Then we have

$$
D_{\mathcal{\zeta}}\left(x^{*}, y^{*}\right) \leq \xi\left(x^{*}, y^{*}, x_{n}, x_{n+1}\right)\left[D_{\mathcal{\zeta}}\left(x^{*}, x_{n}\right)+D_{\mathcal{\zeta}}\left(x_{n}, x_{n+1}\right)+D_{\mathcal{\zeta}}\left(x_{n+1}, y^{*}\right)\right] .
$$

Since

$$
\lim _{n \rightarrow \infty} D_{\xi}\left(x^{*}, x_{n}\right)=0, \lim _{n \rightarrow \infty} D_{\xi}\left(x_{n}, x_{n+1}\right)=0, \lim _{n \rightarrow \infty} D_{\xi}\left(x_{n+1}, y^{*}\right)=0,
$$

we obtain $D_{\xi}\left(x^{*}, y^{*}\right)=0$, so $x^{*}=y^{*}$.

The next result is a version of Kannan's theorem in controlled rectangular b-metric space.

Theorem 5. Let $T: X \rightarrow X$ be a mapping on $D_{\xi}$-complete $C R b$-MS $\left(X, D_{\xi}\right)$, satisfying the following condition: For all $z, t \in X$, there exists $\delta \in\left[0, \frac{1}{2}\right)$ such that

$$
D_{\xi}(T z, T t) \leq \delta\left[D_{\xi}(z, T z)+D_{\xi}(t, T t)\right] .
$$

Additionally, if

$$
\lim _{n \rightarrow \infty} \xi\left(u, T u, x_{n}, x_{n+1}\right) \leq \frac{1}{\delta}
$$

for all $u \in X$, then $T$ has a unique fixed point in $X$.

Proof. Let $\left\{x_{n}\right\}$ be a sequence defined as in Remark 3. From condition (27), we obtain

$$
\begin{gathered}
d\left(x_{n+1}, x_{n}\right) \leq \delta\left[D_{\xi}\left(x_{n}, x_{n+1}\right)+D_{\xi}\left(x_{n-1}, x_{n}\right)\right] \\
\left.d\left(x_{n+1}, x_{n}\right) \leq \frac{\delta}{1-\delta} D_{\xi}\left(x_{n}, x_{n-1}\right)\right]
\end{gathered}
$$

so,

$$
\left.d\left(x_{n+1}, x_{n}\right) \leq \beta^{n} D_{\xi}\left(x_{1}, x_{0}\right)\right]
$$

for all $n \in \mathbb{N}$, where $\beta=\frac{\delta}{1-\delta}$. Additionally, from condition (27), we have

$$
d\left(x_{m}, x_{n}\right) \leq \delta\left[D_{\tilde{\xi}}\left(x_{m-1}, x_{m}\right)+D_{\tilde{\zeta}}\left(x_{n-1}, x_{n}\right)\right] .
$$

Using condition (29), we get

$$
d\left(x_{m}, x_{n}\right) \leq \delta\left[\beta^{m-1} D_{\xi}\left(x_{0}, x_{1}\right)+\beta^{n-1} D_{\xi}\left(x_{0}, x_{1}\right)\right]
$$


for all $m, n \in \mathbb{N}$. Therefore, $\left\{x_{n}\right\}$ is $D_{\tilde{\xi}}$-Cauchy. Let $a^{*}=\lim _{n \rightarrow+\infty} x_{n}$. Since, $X$ is controlled rectangular $b$-metric space we obtain

$$
\begin{aligned}
D_{\xi}\left(a^{*}, T a^{*}\right) \leq & \xi\left(a^{*}, T a^{*}, x_{n}, x_{n+1}\right)\left[D_{\xi}\left(a^{*}, x_{n}\right)+D_{\xi}\left(x_{n}, x_{n+1}\right)+D_{\xi}\left(x_{n+1}, T a^{*}\right)\right] \\
\leq & \xi\left(a^{*}, T a^{*}, x_{n}, x_{n+1}\right)\left[D_{\mathcal{\zeta}}\left(a^{*}, x_{n}\right)+D_{\mathcal{\xi}}\left(x_{n}, x_{n+1}\right)+\right. \\
& \delta\left(D_{\xi}\left(x_{n}, x_{n+1}\right)+D_{\mathcal{\xi}}\left(a^{*}, T a^{*}\right)\right] .
\end{aligned}
$$

Now, from condition (28), we obtain $T a^{*}=a^{*}$. Uniqueness follows from condition (27).

Remark 4. Note that instead of condition (28) in Theorem 5 we can use the condition that $T$ is continuous.

Corollary 1. [15] (see also [16-18]) Let $\left(X, D_{\xi}\right)$ be a complete b-metric space and $T: X \rightarrow X$ such that

$$
D_{\xi}(T s, T r) \leq \lambda D_{\xi}(s, r)
$$

for all $s, r \in X$, where $\lambda \in[0,1)$. Then, $T$ has a unique fixed point in $X$.

Remark 5. As a consequence of Theorem 4, we obtain results for rectangular b-metric space (theorem 2.1 of [6]) and extended b-metric space (corollary 3.3 in [19] and theorem 2 in [20]).

\section{Application in Fractional Differential Type Equation}

In this section, using Theorem 3, we obtain existence and uniqueness solutions of nonlinear fractional differential equation system Caputo type (see, for example, Ref. [21])

$$
\begin{aligned}
& { }^{C} D^{\theta}(\psi(\iota)+\chi(\iota, \psi(\iota)))=0, \iota \in[0,1), \theta<1 \\
& \psi(0)=\psi(1)=0,
\end{aligned}
$$

where $\psi \in C([0,1], \mathbb{R})$ and $C([0,1], \mathbb{R})$ is the set of all continuous functions from $[0,1]$ to $\mathbb{R}, \chi$ : $[0,1] \times \mathbb{R} \rightarrow \mathbb{R}$ is a continuous function, and ${ }^{C} D^{\theta}$ is the Caputo derivative of order $\theta$

$$
{ }^{C} D^{\theta}(\omega(\iota)):=\frac{1}{\Gamma(n-\theta)} \int_{0}^{\iota}(\iota-\kappa)^{n-\theta-1} \omega^{(n)}(\kappa) d \kappa,(n-1<\theta<n),
$$

where $\omega:[0, \infty) \rightarrow \mathbb{R}$ is a continuous function, see [22-24]).

Let $G$ be a Green function for the system (31), for $\alpha<1$, as follows:

$$
G(\iota, \kappa)= \begin{cases}(\iota(1-\kappa))^{\alpha-1}-(\iota-\kappa)^{\alpha-1}, & \text { if } 0 \leq \kappa \leq t \leq 1 \\ \frac{(\iota(1-\kappa))^{\alpha-1}}{\Gamma(\alpha)}, & \text { if } 0 \leq \iota \leq \kappa \leq 1 .\end{cases}
$$

Let us give the first existence result of this section.

Theorem 6. Let the nonlinear fractional differential Equation (31) and $\mu: \mathbb{R} \times \mathbb{R} \rightarrow \mathbb{R}$ be a given function, such that the following assumptions are true:

(i) There exists $\psi_{0} \in(C[0,1], \mathbb{R})$ such that $\mu\left(\psi_{0}(\iota), \int_{0}^{1} T \psi_{0}(\iota) d \iota\right) \geq 0$ for all $\iota \in[0,1]$, where $T$ : $C([0,1], \mathbb{R}) \rightarrow C([0,1], \mathbb{R})$ is defined as follows:

$$
T \psi(\iota)=\int_{0}^{1} G(\iota, \kappa) \chi(\kappa, x(\kappa)) d \kappa
$$


(ii) $|\chi(\iota, \alpha)-\chi(\iota, \beta)| \leq \frac{1}{\sqrt{\tau}} \xi(\alpha, \beta, T \alpha, T \beta)|\alpha-\beta|$ for all $\iota \in[0,1], \tau>1$ and $\alpha, \beta \in \mathbb{R}$ with $\mu(\alpha, \beta) \geq 0$ where $\xi: X^{4} \rightarrow[1, \infty)$;

(iii) for each $\iota \in[0,1]$, if $\left\{\psi_{n}\right\}$ is a sequence in $C([0,1], \mathbb{R})$ such that $\psi_{n} \rightarrow \psi$ in $C([0,1], \mathbb{R})$ and $\mu\left(x_{n}(\iota), x_{n+1}(\iota)\right) \geq 0$ for all $n \in \mathbb{N}$, then $\mu\left(\psi_{n}(t), \psi(t)\right) \geq 0$ for all $n \in \mathbb{N}$.

Then, the Equation (31) has a unique solution.

Proof. Let $X=C([0,1], \mathbb{R})$ endowed with $D_{\xi}: X \times X \rightarrow$ such that

$$
D_{\tilde{\zeta}}(\psi, \varphi)=\|\psi-\varphi\|_{\infty}=\sup _{\iota \in[0,1]}|\psi(\iota)-\varphi(\iota)|^{2}
$$

and $\xi: X^{4} \rightarrow[1, \infty)$ defined by $\xi(\psi, \varphi, T \psi, T \varphi)=\max \{\psi, \varphi, T \psi, T \varphi\}+2$ for any $\psi, \varphi \in X$. Clearly, $\left(X, D_{\xi}\right)$ is a $D_{\tilde{\xi}}$-complete CRb-MS.

It is obvious the problem (31) can be resumed to finding an element $\psi^{*} \in X$ which is a fixed point for the operator $T$.

Let $\psi, \varphi \in X$, such that $\mu(\psi(\iota), \varphi(\iota)) \geq 0$ for all $\iota \in[0,1]$. By $(i)$ and (ii), we have true the following inequalities:

$$
\begin{aligned}
& |T \psi(\iota)-T \varphi(\iota)|^{2}=\left|\int_{0}^{1} G(\iota, \kappa)[\chi(\kappa, \psi(\kappa))-\chi(\kappa, \varphi(\kappa))] d \kappa\right|^{2} \\
& \leq\left(\int_{0}^{1} G(\iota, \kappa) d \kappa\right)^{2} \int_{0}^{1}|\chi(\kappa, \psi(\kappa))-\chi(\kappa, \varphi(\kappa))|^{2} d \kappa \\
& \leq \frac{1}{\tau} \int_{0}^{1} \xi^{2}(\psi(\kappa), \varphi(\kappa), T \psi(\kappa), T \varphi(\kappa))|\psi(\kappa)-\varphi(\kappa)|^{2} d \kappa\left(\int_{0}^{1} G(\iota, \kappa) d \kappa\right)^{2} .
\end{aligned}
$$

Let $\xi(\psi(\kappa), \varphi(\kappa), T \psi(\kappa), T \varphi(\kappa))=\max _{\kappa \in[0,1]}\{\psi(\kappa), \varphi(\kappa), T \psi(\kappa), T \varphi(\kappa)\}+2=\gamma+2=\sqrt{\lambda}$ be such that $\lambda<\tau$.

Then, we have

$$
|T x(\iota)-T y(\iota)|^{2} \leq \frac{\lambda}{\tau}|\psi(\iota)-\varphi(\iota)|^{2}\left(\int_{0}^{1} G(\iota, \kappa) d \kappa\right)^{2}
$$

Then, taking supremum in both sides, for every $\psi, \varphi \in X$ with $\mu(\psi(\iota), \varphi(\iota))>0$ and $\iota \in[0,1]$, we obtain

$$
D_{\xi}(\psi, \varphi)=\|T \psi-T \varphi\|_{\infty} \leq \frac{\lambda}{\tau}\|\psi-\varphi\|_{\infty}=\frac{\lambda}{\tau} D_{\tilde{\xi}}(\psi, \varphi) .
$$

It is easy to check that

$$
\prod_{i=1}^{k} \xi\left(\psi_{m+k-i}(\kappa), \psi_{n+k-i}(\kappa), \psi_{m+k+1-i}(\kappa), \psi_{n+k+1-i}(\kappa)\right)=\prod_{i=1}^{k} \gamma_{i}<+\infty,
$$

and

$$
\xi\left(\psi_{m}(\kappa), \psi_{n}(\kappa), \psi_{m+k}(\kappa), \psi_{n+k}(\kappa)\right)<+\infty,
$$

for all $k, m, n \geq n_{0}$, with $n_{0} \in \mathbb{N}$.

For $0<\delta=\frac{1}{\tau}<1$ and $\lambda<\tau$, we conclude that all the conditions of the Theorem 3 are accomplished. Then, the system (31) has a unique solution. 
Let us consider the following type of integral equation

$$
x(\iota)=q(\iota)+\int_{0}^{t} P(\iota, \kappa) g(\kappa, x(\kappa)) d \kappa, \iota \in[0,1], x(\iota) \in X,
$$

where $g(\iota, x(\iota)):[0,1] \times \mathbb{R} \rightarrow \mathbb{R}, q(\iota):[0,1] \rightarrow \mathbb{R}$ are two bounded continuous functions and $P:[0,1] \times[0,1] \rightarrow[0, \infty)$ is a function such that $P(\iota, \cdot) \in L^{1}([0,1])$ for all $\iota \in[0,1]$.

Then, our aim is to establish the existence of a solution for the previous integral Equation (34) using Theorem 4. In this frame, let us give the following result.

Theorem 7. Let $X=C([0,1], \mathbb{R})$ be the set of all continuous real-valued functions defined on $[0,1]$. Let $T$ : $X \rightarrow X$ be an operator defined by:

$$
T x(\iota)=q(\iota)+\int_{0}^{\iota} P(\iota, \kappa) g(\kappa, x(\kappa)) d \kappa, \iota \in[0,1], x(\iota) \in X .
$$

Suppose the following conditions hold:

(i) The functions $q(\iota):[0,1] \rightarrow \mathbb{R}$ and $g(\iota, x(\iota)):[0,1] \times \mathbb{R} \rightarrow \mathbb{R}$ are continuous;

(ii) $P:[0,1] \times[0,1] \rightarrow[0, \infty)$ is a function such that $P(\iota, \cdot) \in L^{1}([0,1])$ for all $\iota \in[0,1]$ and, for every $\kappa \in[0,1]$, we have:

$$
\left\|\int_{0}^{l} P(\iota, \kappa) d \kappa\right\|<1 .
$$

(iii) $|g(\kappa, x(\kappa))-g(\kappa, y(\kappa))| \leq \frac{1}{\sqrt{\alpha}}|x(\kappa)-y(\kappa)|$, for all $x, y \in X$ and $\alpha>e^{2 \tau \iota}$.

Then, the Equation (34) has a unique solution.

Proof. Let $X=C([0,1], \mathbb{R})$ endowed with the Bielecki norm

$$
D_{\xi}=\|x\|_{B}=\sup _{\iota \in[0,1]}|x(\iota)| e^{-\tau \iota} \text { with } \tau>1
$$

and $\xi: X^{4} \rightarrow[1, \infty)$ defined by $\xi(x, y, T x, T y)=\max \{x, y, T x, T y\}+2$ for any $x, y \in X$. It is easy to

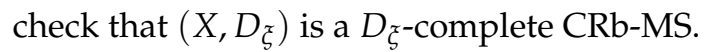

Then, our problem (34) can be resumed to finding an element $x^{*} \in X$ which is a fixed point for the operator $T$.

We have the estimation

$$
\begin{aligned}
|T x(\iota)-T y(\iota)|^{2} & \leq\left|\int_{0}^{\iota}[P(\iota, \kappa) g(\kappa, x(\kappa))-P(\iota, \kappa) g(\kappa, y(\kappa))] d \kappa\right|^{2} \\
& \leq \int_{0}^{\iota}|P(\iota, \kappa)[g(\kappa, x(\kappa))-g(\kappa, y(\kappa))]|^{2} d \kappa \\
& \leq\left(\int_{0}^{\iota} P(\iota, \kappa) d \kappa\right)^{2} \int_{0}^{\iota}|[g(\kappa, x(\kappa))-g(\kappa, y(\kappa))]|^{2} d \kappa \\
& \leq \frac{1}{\alpha} \int_{0}^{\iota}|x(\kappa)-y(\kappa)|^{2} d \kappa\left(\int_{0}^{\iota} P(\iota, \kappa) d \kappa\right)^{2} \\
& =\frac{e^{2 \tau \iota}}{\alpha e^{-2 \tau \iota}} \int_{0}^{\iota}|x(\kappa)-y(\kappa)|^{2} e^{-2 \tau \iota} d \kappa\left(\int_{0}^{\iota} P(\iota, \kappa) e^{-\tau \iota} d \kappa\right)^{2} .
\end{aligned}
$$


Thus, we obtain

$$
|T x(\iota)-T y(\iota)|^{2} \leq \frac{e^{2 \tau \iota}}{\alpha e^{-2 \tau \iota}} \int_{0}^{\iota}|x(\kappa)-y(\kappa)|^{2} e^{-2 \tau \iota} d \kappa\left(\int_{0}^{\iota} P(\iota, \kappa) e^{-\tau \iota} d \kappa\right)^{2} .
$$

Taking supremum in the inequality (36), we have

$$
\left[\sup _{\iota \in[0,1]}|T x(\iota)-T y(\iota)| e^{-\tau \iota}\right]^{2} \leq \frac{1}{\alpha e^{-2 \tau \iota}}\left[\sup _{\iota \in[0,1]}|x(\iota)-y(\iota)| e^{-\tau \iota}\right]^{2}\left(\int_{0}^{\iota} \sup _{\iota \in[0,1]} P(\iota, \kappa) e^{-\tau \iota} d \kappa\right)^{2} .
$$

Using the hypothesis (ii), we have

$$
D_{\tilde{\zeta}}(x, y)=\|T x-T y\|_{B} \leq \frac{1}{\sqrt{\alpha} e^{-\tau \iota}}\|x-y\|_{B}=\frac{1}{\sqrt{\alpha} e^{-\tau \iota}} D_{\tilde{\xi}}(x, y) .
$$

It is easy to check that $\xi\left(x_{m}(\kappa), x_{n}(\kappa), x_{m+k}(\kappa), x_{n+k}(\kappa)\right)<+\infty$, for all $k, m, n \geq n_{0}$, with $n_{0} \in \mathbb{N}$. Then, for $0<\delta=\frac{1}{\sqrt{\alpha} e^{-\tau \iota}}<1$, we have true all the conditions of Theorem 4 . In this condition, we can conclude that Equation (34) has a unique solution.

\section{Conclusions}

In this paper, we gave an affirmative answer to an open question posed recently by Mlaiki et al. [Adv. Math. Phys., 2020]. As a consequence of our results, we get some known results in the literature. We also gave an application of our results to the existence of a solution of nonlinear fractional differential equations. We believe that our results will have an impact on other researchers in this field.

Author Contributions: Investigation, R.G., A.B., S.B., Z.D.M., N.M. and L.G.; Methodology, R.G., Z.D.M., L.G.; Software, Z.D.M.; Supervision, R.G., Z.D.M., and L.G. All authors have read and agreed to the published version of the manuscript.

Funding: This research received no external funding.

Acknowledgments: The authors are thankful to the Deanship of Scientific Research at Prince Sattam bin Abdulaziz University, Al-Kharj, Kingdom of Saudi Arabia, for supporting this research.

Conflicts of Interest: The authors declare no conflict of interest.

\section{References}

1. Fréchet, M. Sur quelques points du calcul fonctionnel. Rendiconti del Circolo Matematico di Palermo (1884-1940) $1906,22,1-72$.

2. Hausdorff, F. Grundzuge der Mengenlehre. Monatshefte für Mathematik und Physik 1915, 26, A34-A35.

3. Banach, S. Sur les opérations dans les ensembles abstraits et leur application aux équations intégrales. Fundam. Math. 1922, 3, 133-181. [CrossRef]

4. Bakhtin, I.A. The contraction mapping principle in quasi-metric spaces. Funct. Anal. 1989, 30, 26-37.

5. Branciari, A. A fixed point theorem of Banach-Caccioppoli type on a class of generalized metric spaces. Publ. Math. 2000, 57, 31-37.

6. George, R.; Radenović, S.; Rashma, K.P.; Shukla, S. Rectangular $b$-metric spaces and contraction principals. J. Nonlinear Sci. Appl. 2015, 8, 1005-1013. [CrossRef]

7. Mlaiki, N.; Aydi, H.; Souayah, N.; Abdeljawad, N. Controlled Metric Type Spaces and the Related Contraction Principle. Mathematics 2018, 6, 194. [CrossRef]

8. Abdeljawad, T.; Mlaiki, N.; Aydi, H.; Souayah, N. Double Controlled Metric Type Spaces and Some Fixed Point Results. Mathematics 2018, 6, 320. [CrossRef]

9. Abdeljawad, T.; Karapınar, E.; Panda, S.K.; Mlaiki, N. Solutions of boundary value problems on extended-Branciari b-distance. J. Inequal. Appl. 2020, 2020, 103. [CrossRef] 
10. Shatanawi, W.; Abodayeh, K.; Mukhemer, A. Some fixed point theorems in extended b-metric spaces. U.P.B. Sci. Bull. Ser. A 2018, 80, 71-78.

11. Ali, M.U.; Kamran, T.; Postolache, M. Solution of Volterra integral inclusion in $b$-metric spaces via new fixed point theorem. Nonlinear Anal. Model. Control 2017, 22, 17-30. [CrossRef]

12. Kamran, T.; Postolache, M.; Ali, M.U.; Kiran, Q. Feng and Liu type F-contraction in $b$-metric spaces with application to integral equations. J. Math. Anal. 2016, 7, 18-27.

13. Abodayeh, K.; Karapınar, E.; Pitea, A.; Shatanawi, W. Hybrid Contractions on Branciari Type Distance Spaces. Mathematics 2019, 7, 994. [CrossRef]

14. Mlaiki, N.; Hajji, M.; Abdeljawad, T. A New Extension of the Rectangular b-Metric Spaces. Adv. Math. Phys. 2020, 7. [CrossRef]

15. Czerwik, S. Contraction mappings in b-metric spaces. Acta Math. Inform. Univ. Ostrav. 1993, 1, 5-11.

16. Dung, N.V.; An, T.V.; Hang, V.T.L. Remarks on Frink's metrization technique and applications. Fixed Point Theory 2019, 20, 157-176. [CrossRef]

17. Mitrović, Z.D. A note on a Banach's fixed point theorem in $b$-rectangular metric space and b-metric space. Math. Slovaca. 2018, 68, 1113-1116. [CrossRef]

18. Mitrović, Z.D. Fixed point results in $b$-metric space. Fixed Point Theory 2019, 20, 559-566. [CrossRef]

19. Mitrović, Z.D.; Işik, H.; Radenović, S. The new results in extended $b$-metric spaces and applications. Int. J. Nonlinear Anal. Appl. 2020, 11, 473-482.

20. Kamran, T.; Samreen, M.; Qurat, U.A. A generalization of $b$-Metric space and some fixed point theorems. Mathematics 2017, 5, 19. [CrossRef]

21. Podlubny, I. Fractional Differential Equations; Academic Press: San Diego, CA, USA, 1999.

22. Kilbas, A.A.; Srivastava, M.H.; Trujillo, J.J. Theory and Application of Fractional Differential Equations. In North Holland Mathematics Studies; Elsevier: Amsterdam, The Netherlands, 2006; Volume 204.

23. Samko, S.G.; Kilbas, A.A.; Marichev, O.I. Fractional Integral and Derivative; Gordon Breach: London, UK, 1993.

24. Vlase, S.; Marin, M.; Öchsner, A.; Scutaru, M.L. Motion equation for a flexible one-dimensional element used in the dynamical analysis of a multibody system. Contin. Mech. Thermodyn. 2019, 31, 715-724. [CrossRef]

Publisher's Note: MDPI stays neutral with regard to jurisdictional claims in published maps and institutional affiliations.

(C) 2020 by the authors. Licensee MDPI, Basel, Switzerland. This article is an open access article distributed under the terms and conditions of the Creative Commons Attribution (CC BY) license (http://creativecommons.org/licenses/by/4.0/). 